\title{
Gait Changes Following Robot-Assisted Gait Training in Children With Cerebral Palsy
}

\section{Dragana ŽARKOVIĆ ${ }^{1}$, Monika ŠORFOVÁ ${ }^{1}$, James J. TUFANO ${ }^{2}$, Patrik KUTÍLEK ${ }^{3}$, Slávka VÍTEČKOVÁ ${ }^{4}$, David RAVNIK ${ }^{5}$, Katja GROLEGER-SRŠEN ${ }^{6}$, Imre CIKAJLO ${ }^{7}$, Jakub OTÁHAL ${ }^{8}$}

${ }^{1}$ Department of Anatomy and Biomechanics, Faculty of Physical Education and Sport, Charles University, Prague, Czech Republic, ${ }^{2}$ Department of Physiology and Biochemistry, Faculty of Physical Education and Sport, Charles University, Prague, Czech Republic, ${ }^{3}$ Department of Natural Sciences, Faculty of Biomedical Engineering, Czech Technical University in Prague, Kladno, Czech Republic, ${ }^{4}$ Department of Biomedical Informatics, Faculty of Biomedical Engineering, Czech Technical University in Prague, Kladno, Czech Republic, ${ }^{5}$ Department of Physiotherapy, Faculty of Health Sciences, University of Primorska, Izola, Republic of Slovenia, ${ }^{6}$ Children's Rehabilitation Department, University Rehabilitation Institute, Faculty of Medicine, University of Ljubljana, Ljubljana, Republic of Slovenia, ${ }^{7}$ Laboratory of Clinical Kinesiology, Faculty of Medicine, University Rehabilitation Institute, University of Ljubljana, Ljubljana, Republic of Slovenia, ${ }^{8}$ Department of Developmental Epileptology, Institute of Physiology, Prague, Czech Republic

Received April 16, 2021

Accepted November 15, 2021

\begin{abstract}
Summary
This study investigated changes of gait pattern induced by a 4-week robot-assisted gait training (RAGT) in twelve ambulatory spastic diparesis children with cerebral palsy (CP) aged $10.4 \pm 3.2$ years old by using computerized gait analysis (CGA). Pre-post intervention CGA data of children with CP was contrasted to the normative data of typically developing children by using cross-correlation and statistically evaluated by a Wilcoxon test. Significant pre-post intervention changes $(p<0.01)$ include: decreased muscle activity of biceps femoris, rectus femoris, and tibialis anterior; a decrease in range of internal hip joint rotation, higher cadence, step length, and increased stride time. This study suggests that RAGT can be used in muscle reeducation and improved hip joint motion range in ambulatory children with $\mathrm{CP}$.
\end{abstract}

\section{Key words}

Cerebral palsy • Spastic diparesis • Gait cycle • Computerized gait analysis • Robot-assisted gait training

\section{Corresponding author}

D. Žarković, Department of Anatomy and Biomechanics, Faculty of
Physical Education and Sport, Charles University, José Martího 269/31, 162 52, Prague, Czech Republic. E-mail: dragana.z@seznam.cz

\section{Introduction}

Cerebral palsy

Cerebral palsy (CP) is a developmental disability that was first described in the 1840 s, yet continues to be one of the most frequent pediatric disabilities with an incidence of 2-3 per 1000 children (Bax et al. 2005, Panteliadis 2004). Most physiotherapy approaches aim to improve movement quality and develop skills necessary to carry out activities of daily living, which often include tasks such as standing and walking (Novak 2014).

\section{Robot-assisted gait training (RAGT)}

Although manual assistance can be used to aid children with CP, RAGT allows for more advanced and customizable gait rehabilitation programs. RAGT was introduced 15 years ago and consists of bilateral robotic orthoses, body-weight support (BWS), and a treadmill. 
Being a computerized system, it is possible to adjust the amount of BWS to maintain extended posture and provide accurate loading of the lower limbs. The robotic orthoses guide a patient's leg movements throughout repeatable predefined trajectories of the hip- and kneejoints in the sagittal plane, and foot supports maintain passive ankle dorsiflexion, which can aid CP children while walking (Colombo et al. 2000, Riener et al. 2005, Meyer-Heim et al. 2007). The main aim of RAGT is to improve the motor learning process through repetitive stimulation of gait accompanied by audio-visual feedback (Schuler et al. 2011). RAGT provides a simplified and safe therapeutic environment that allows for prolonged training duration with many repetitions of steps while inducing a reproducible, kinematically consistent, symmetrical gait pattern (Colombo et al. 2000). RAGT seems to be a promising method for children with $\mathrm{CP}$ in improved selective voluntary motor control (Žarković et al. 2020), increased muscle activity, and inter limb symmetry (Schuler et al. 2011, Schuler et al. 2013, Bonikowski et al. 2012), restricted passive joint range of motion (Vrečar et al. 2013, Žarković et al. 2020), and improved gross motor abilities including improved spatiotemporal gait parameters (Meyer-Heim et al. 2007, Borggraeffe et al. 2010, Knecht et al. 2010, Žarković et al. 2020). Despite that, it is still unknown whether functional motor improvements following RAGT can also contribute to the improved quality of gait patterns. Therefore, the purpose of this experimental study was to investigate changes in gait pattern following RAGT as a monotherapy in ambulatory spastic diparesis children with CP. Knowing that motor learning is critical for neuroplasticity and that children with $\mathrm{CP}$ can improve motor function with practice (Hemayattalab et al. 2010, Gordon et al. 2012, Hemayattalab et al. 2013), we hypothesized that RAGT can induce physiological gait changes in lower limbs that can be comparable to the healthy children.

\section{Methods}

\section{Ethics Committee approval}

The study received ethics committee approvals from participating institutions (No. 120/2015). The parents of the children were informed of the study procedures, risks, and benefits, and provided written informed consent before their children participating in the study.

\section{Children with CP}

Inclusion criteria were: $\mathrm{CP}$ spastic diparesis with toe walking pattern; Gross Motor Function Classification (GMFCS) I-III; ability to walk independently for at least short distances; age range 5-17 years; femur length at least $21 \mathrm{~cm}$ to fit in orthoses of RAGT device; ability to communicate fear, pain, or discomfort; ability to follow simple instructions; no botulinum toxin in the last 3 months before RAGT; no orthopedic surgical intervention in the last 12 months; no severe contractures; and ability to attend 20 RAGT sessions scheduled in 20 consecutive weekdays (Vrečar et al. 2013, Wallard et al. 2018).

\section{Definition of limb impairment}

Before the RAGT intervention, an experienced pediatric physiotherapist performed in all children a passive joint range of motion (PROM) goniometry evaluation of lower limbs (Janda et al. 1993), and the Selective Control Assessment for the Lower Extremity (SCALE) (Fowler et al. 2009). SCALE and PROM revealed "more impaired limb" (MIL) and "less impaired limb" (LIL), as well as evaluations excluded patients with severe joint contractures (Fowler et al. 2009, Syczewska and Świecicka 2016). These evaluations had an informatory purpose that was summarized in section results.

\section{Data collection}

Computerized gait analysis (CGA)

The CGA included a comprehensive 3D gait analysis consisting of joint kinematics, kinetics, sEMG, and spatiotemporal parameters. The CGA evaluation was performed by 2 well-trained physiotherapists and 2 biomechanics experts. First, the skin was gently abraded, and multi-purpose monitoring electrodes were placed on the following muscles bilaterally according to the SENIAM recommendations (Hermens et al. 1999): tibialis anterior (TA), medial gastrocnemius (MG), rectus femoris (RF), and biceps femoris (BF). A neutral reference electrode was attached to the tensor fascia latae muscle (Schuler et al. 2011). Subsequently, 17 reflective markers (Vicon, Oxford Metrics, Oxford, UK) were attached directly to the skin to ensure a fixed and precise position. Markers were attached bilaterally to the following body areas concerning the Vicon Plug-in-gait model (Davis et al. 1991, Dixon et al. 2014, Flux et al. 2020): second metatarsal joint; middle of the Achilles tendon; malleolus lateralis; center of the tibia; lateral femoral epicondyle; lateral side of the thigh; spina iliaca 
anterior superior (SIAS); L5; Th10; sternum. All children with $\mathrm{CP}$ were told to walk barefoot in the pre-designed 10-meter flat-surface pathway to adapt to space. Afterward, children with $\mathrm{CP}$ were appealed to walk barefoot without walking aids at preferred speed and according to physical capacities for a minimum of ten trials on the same pre-designed 10-meter flat-surface pathway in the gait laboratory. Kinematics, kinetics, and sEMG data were collected and recorded simultaneously. 3D kinematic data was recorded by using a 6-camera VICON system (0.3 MPix VICON, Oxford Metrics, Oxford, UK) with a sampling frequency of $50 \mathrm{~Hz}$ until the course of three completed trials was obtained. Kinetics was recorded by using AMTI force plates (AMTI OR6, Advanced Mechanical Technology Inc., Watertown, MA) at $1 \mathrm{kHz}$ sampling frequency. Muscle activity was recorded with an 8-channel sEMG sampling frequency at $1 \mathrm{kHz}$ (Noraxon TeleMyo 2400T, Noraxon U.S.A. Inc.).

\section{RAGT intervention}

The RAGT device Lokomat Pro (Hocoma AG, Volketswil, Switzerland) was used. The intervention consisted of twenty sessions scheduled for 20 consecutive workdays with a minimum duration of 30 and up to a maximum of 45 min (Vrečar et al. 2013, Wallard et al. 2017). The treadmill speed was synchronized with the movements of the robotic orthoses and set to a comfortable walking speed of every child individually. These parameters were set by following the child's ability to walk at a certain speed, follow the augmented feedback and maintain an upright posture. All children wore shoes during the RAGT. At the beginning of RAGT program, all children had an initial level of BWS set to $50 \%$ of body weight (Schuler et al. 2013). The BWS was further decreased for every child individually until the knee started to collapse into flexion during the stance phase due to the increased load of body weight. All children walked with augmented biofeedback (Schuler et al. 2011, Schuler et al. 2013, Wallard et al. 2017). For consistency, the same physiotherapist was present at every RAGT session to follow the progression and encourage the child to walk actively and keep an extended posture (Fig. 1).

\section{Data evaluation}

\section{Data processing}

Raw CGA data obtained from overground gait was high-pass filtered by the VICON system (VICON Nexus 1.8.3.) to enable analog data sampling with $1 \mathrm{kHz}$, and subsequently filtered with a $4^{\text {th }}$ order low-pass Butterworth filter with a cut off frequency of $20 \mathrm{~Hz}$
(Kadaba et al. 1989, Baker 2013). The data was normalized and the Vicon Plug-in-Gait model was used to generate kinematic and kinetic data (Davis et al. 1991). Joint angles were calculated based on the 3D coordinates of markers. Internal joint moments and power were calculated based on joint kinematics and ground reaction forces recorded using force plates (Kadaba et al. 1989, MacWilliams et al. 2003, Baker 2013). Force plates measured ground reaction forces and center of pressure (COP) trajectory (Baker 2013). VICON Nexus 1.8.3. and Polygon 3.5.1. softwares (VICON, Oxford Metrics, Oxford, UK) were used to define the gait cycles, spatiotemporal parameters, joint angles, internal joint moments, and power. sEMG data was processed by MyoResearch XP 1.07 Master Edition software (Noraxon Inc., Scottsdale/USA). Raw sEMG signals were highpass filtered with a bi-directional zero-lag Butterworth at a cut-off frequency of $10 \mathrm{~Hz}$, rectified, and smoothed with a time window of $100 \mathrm{~ms}$ to create the linear envelope. The sEMG data was normalized to the maximum EMG recorded during the gait cycle (Fung et al. 1989, Burden and Bartlett 1999, Burden et al. 2003, Bojanic et al. 2011, Aurich-Schuler 2017, Ricklin et al. 2018). As subjects walked for a minimum of ten trials, gait cycles were identified in each trial. Heel strike and toe-off markers were set automatically by the software program and adjusted manually if necessary. The gait cycle starts and ends with a heel strike of the same lower extremity (Perry 2010, Baker 2013). Within the cycles, the mean value of these trials was calculated to obtain 1 gait cycle and separate gait phases. The gait cycle was represented by 51 evenly spaced samples $(0-100 \%$ in $2 \%$ steps) that were marked as initial contact (IC), loading response (LR), midstance (MST), terminal stance (TS), pressing (PSW), initial swing (ISW), midswing (MSW), terminal swing (TSW) (Perry et al. 2010). This study aimed to explore whether RAGT can induce physiological gait changes in lower limbs that will be comparable to healthy children. Therefore, all collected CGA variables were contrasted to the normative data (Sutherland 2002, Hof et al. 2005, Winter et al. 2009) assessed in typically developing children, and differences in individual phases of the gait cycle (Perry 2010) were examined to explain the pathological gait mechanism and compensatory movements. Normative data represent an integral part that is built in VICON and Myoresearch softwares. Subsequently, data was used for statistical evaluation by using a custom-written MatLab program (MatLab software processes, MatLab R2010b, Mathworks, Inc., Natick, MA, USA). 


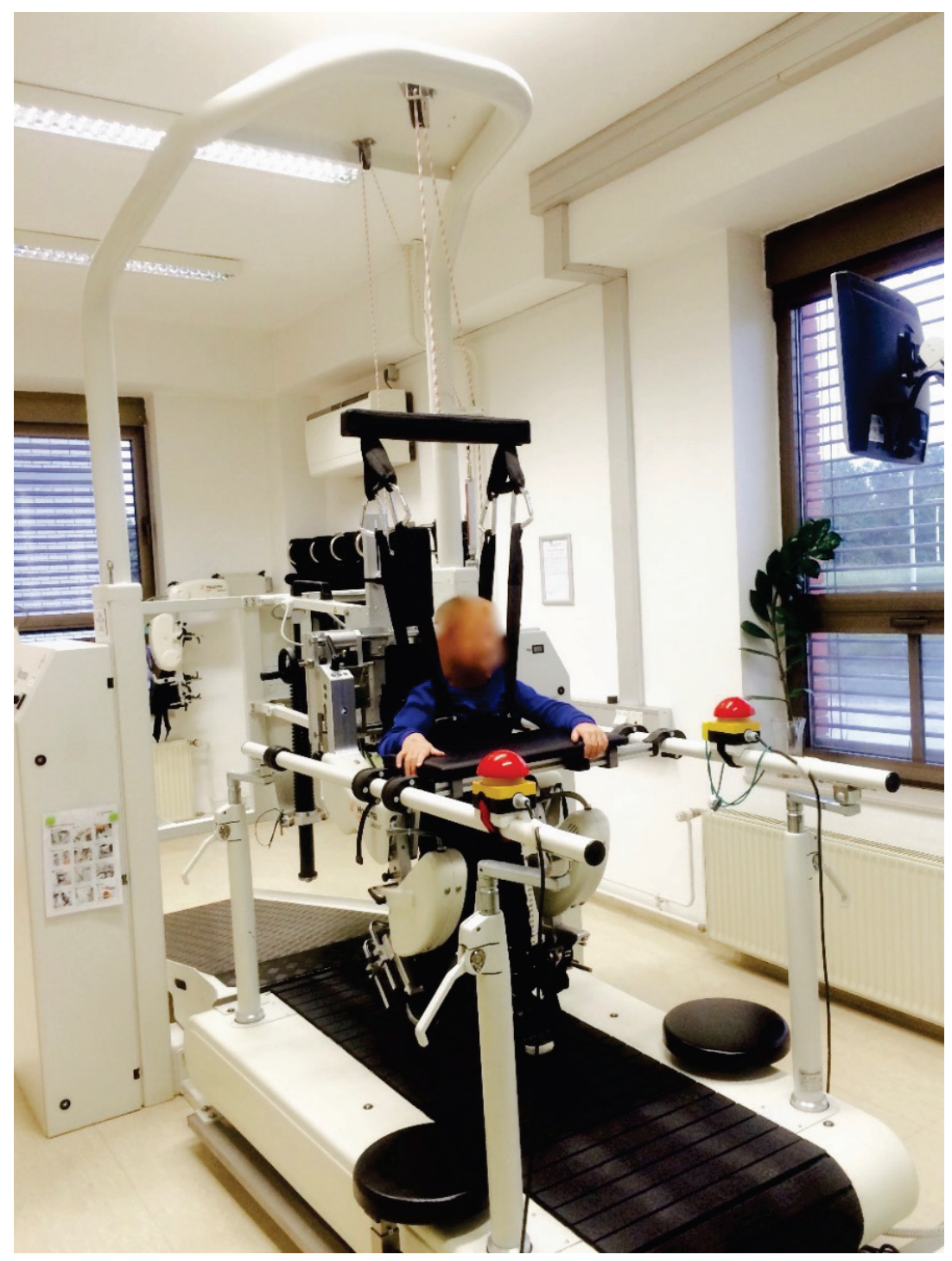

Fig. 1. A 5-year-old boy with spastic diparesis during RAGT using the Lokomat Pro.

\section{Statistical evaluation}

Comprehensive CGA resulted in a statistical evaluation of 43 variables embracing sEMG; motion range of thorax, pelvis, hip, knee, and ankle joints in all three planes; joint moments in sagittal/ frontal planes and power of hip, knee, and ankle joints; center of pressure, center of mass and ground reaction forces in all three planes. First, the deviation of $\mathrm{CP}$ signals from the normative values was calculated by cross-correlation for every variable and particular phases of the gait cycle. It was performed for LIL and MIL separately pre- and postintervention. The statistical evaluation was performed to compare the pre- and post-intervention conditions of children with CP. The Shapiro-Wilk test was used to verify data normality. As normal data distribution has been rejected at the 0.05 significance level, the nonparametric Wilcoxon sign rank test was used for further statistical calculation of each variable and gait cycle phase separately ( 0.05 significance level). The calculation was completed by Bonferroni correction $(\mathrm{p}<0.01)$, median values and effect sizes of cross-correlation coefficients (Cohen 1988).

\section{Results}

\section{Children with $C P$}

Twelve CP children with spastic diparesis ( 2 girls; 10 boys) with apparent equinus gait pattern; aged $10.4 \pm 3.2$ years; GMFCS I-III; with decreased selective voluntary motor control of lower extremities as per SCALE evaluation (total score MIL 5, total score LIL 7 out of max. score 10), and with hip-knee-ankle joint contractures $\left(\leq 10^{\circ}\right.$ for both extremities) completed the RAGT program. The program was well-tolerated by all of the children with $\mathrm{CP}$ and no adverse events were reported. 


\section{CGA results}

Significant improvements contrasted to the normative data were found in bilaterally decreased activity of BF, RF, TA muscles and decreased internal hip joint rotation. There were no significant changes in kinetic variables. Spatiotemporal parameters showed increased cadence and step length, and decrease in time needed for double support and stride. A detailed summary of results is shown in Tables 1-3.

Table 1. Table shows an overview of Wilcoxon sign rank tests for CGA variables with corrected $p$-value, median values and effect sizes of cross-correlation coefficients. Statistically significant results $(p \leq 0.01)$ are bolded and marked with an asterisk $(*)$.

\begin{tabular}{|c|c|c|c|c|c|c|c|c|}
\hline \multirow{3}{*}{$s E M G$} & \multicolumn{8}{|c|}{ Gait cycle phases } \\
\hline & \multicolumn{8}{|c|}{ p-value after Bonferroni correction/effect size of cross-correlation coefficient } \\
\hline & IC & LR & MST & TS & PSW & ISW & MSW & TSW \\
\hline BF MIL & $<0.01 / 0.54$ & $<0.01 / 0.52$ & $<0.01 * / 0.56$ & $<0.01 * / 0.57$ & $<0.01 * / 0.56$ & $<0.01 / 0.51$ & $<0.01 / 0.52$ & $<0.01 * / 0.56$ \\
\hline BF LIL & $<0.01 / 0.50$ & $<0.01 / 0.47$ & $<0.01 / 0.52$ & $<0.01 * / 0.57$ & $<0.01 / 0.36$ & $<0.01 \% / 0.62$ & $<0.01 / 0.52$ & $<0.01 * / 0.57$ \\
\hline$R F M I L$ & $<0.01 * / 0.59$ & $<0.01 * / 0.57$ & $<0.01 * / 0.62$ & $<0.01 * / 0.59$ & $<0.01 * / 0.62$ & $<0.01 / 0.51$ & $<0.01 * / 0.56$ & $<0.01 * / 0.60$ \\
\hline RF LIL & $<0.01 * / 0.59$ & $<0.01 * / 0.60$ & $<0.01 * / 0.60$ & $<0.01 * / 0.62$ & $>0.01 / 0.20$ & $<0.01 / 0.43$ & $<0.01 / 0.43$ & $<0.01 / 0.51$ \\
\hline$M G M I L$ & $<0.01 / 0.46$ & $<0.01 / 0.35$ & $<0.01 / 0.43$ & $<0.01 / 0.44$ & $<0.01 / 0.43$ & $<0.01 / 0.35$ & $<0.01 / 0.48$ & $<0.01 / 0.44$ \\
\hline$M G L I L$ & $<0.01 / 0.39$ & $>0.01 / 0.36$ & $>0.01 / 0.35$ & $<0.01 / 0.41$ & $>0.01 / 0.38$ & $<0.01 / 0.52$ & $<0.01 / 0.52$ & $<0.01 / 0.40$ \\
\hline TA MIL & $<0.01 / 0.46$ & $<0.01 / 0.48$ & $<0.01 / 0.35$ & $<0.01 / 0.38$ & $<0.01 / 0.43$ & $<0.01 / 0.40$ & $<0.01 / 0.28$ & $<0.01 / 0.43$ \\
\hline TA LIL & $<0.01 \% / 0.59$ & $<0.01 * / 0.62$ & $<0.01 * / 0.60$ & $<0.01 * / 0.62$ & $<0.01 / 0.54$ & $<0.01 / 0.54$ & $<0.01 / 0.44$ & $<0.01 / 0.52$ \\
\hline Kinematics & IC & LR & MST & TS & PSW & ISW & MSW & TSW \\
\hline Pelvic tilt MIL & $>0.01 / 0.19$ & $>0.01 / 0.17$ & $>0.01 / 0.11$ & $>0.01 / 0.16$ & $>0.01 / 0.17$ & $>0.01 / 0.20$ & $>0.01 / 0.20$ & $>0.01 / 0.20$ \\
\hline Pelvic tilt LIL & $>0.01 / 0.19$ & $>0.01 / 0.19$ & $>0.01 / 0.19$ & $>0.01 / 0.20$ & $>0.01 / 0.17$ & $>0.01 / 0.12$ & $>0.01 / 0.14$ & $>0.01 / 0.19$ \\
\hline $\begin{array}{l}\text { Pelvic obliquity } \\
\text { MIL }\end{array}$ & $1 / 0$ & $>0.01 / 0.01$ & $>0.01 / 0.20$ & $>0.01 / 0.35$ & $<0.01 /-0.44$ & $>0.01 /-0.25$ & $>0.01 /-0.04$ & $>0.01 /-0.04$ \\
\hline $\begin{array}{l}\text { Pelvic obliquity } \\
\text { LIL }\end{array}$ & $<0.01 / 0.43$ & $>0.01 /-0.32$ & $>0.01 /-0.19$ & $>0.01 / 0.01$ & $>0.01 / 0.06$ & $>0.01 / 0.17$ & $>0.01 / 0.35$ & $<0.01 / 0.43$ \\
\hline $\begin{array}{l}\text { Pelvic rotation } \\
\text { MIL }\end{array}$ & $>0.01 /-0.09$ & $>0.01 /-0.08$ & $>0.01 /-0.22$ & $>0.01 / 0.33$ & $>0.01 / 0.28$ & $>0.01 / 0.14$ & $>0.01 / 0.04$ & $>0.01 / 0.03$ \\
\hline $\begin{array}{l}\text { Pelvic rotation } \\
\text { LIL }\end{array}$ & $>0.01 / 0.36$ & $>0.01 / 0.28$ & $>0.01 / 0.19$ & $>0.01 /-0.09$ & $>0.01 /-0.01$ & $>0.01 /-0.11$ & $>0.01 /-0.24$ & $>0.01 /-0.25$ \\
\hline $\begin{array}{l}\text { Hip flexion/ } \\
\text { extension MIL }\end{array}$ & $>0.01 / 0.04$ & $>0.01 / 0.04$ & $>0.01 / 0.12$ & $>0.01 / 0.09$ & $>0.01 /-0.20$ & $>0.01 / 0.28$ & $>0.01 / 0.25$ & $\begin{array}{c}>0.01 / 0.16 \\
3\end{array}$ \\
\hline $\begin{array}{l}\text { Hip flexion/ } \\
\text { extension LIL }\end{array}$ & $>0.01 / 0.22$ & $>0.01 / 0.25$ & $>0.01 / 0.33$ & $<0.01 / 0.44$ & $>0.01 /-0.24$ & $>0.01 / 0.36$ & $>0.01 / 0.36$ & $>0.01 / 0.28$ \\
\hline $\begin{array}{l}\text { Hip abduction/ } \\
\text { adduction MIL }\end{array}$ & $>0.01 /-0.30$ & $>0.01 / 0.24$ & $>0.01 / 0.33$ & $>0.01 / 0.22$ & $>0.01 /-0.28$ & $\begin{array}{c}>0.01 /- \\
0.25 \\
\end{array}$ & $\begin{array}{c}>0.01 /- \\
0.22\end{array}$ & $>0.01 /-0.24$ \\
\hline $\begin{array}{l}\text { Hip abduction/ } \\
\text { adduction LIL }\end{array}$ & $>0.01 / 0.28$ & $>0.01 /-0.28$ & $>0.01 /-0.14$ & $>0.01 /-0.11$ & $>0.01 /-0.08$ & $>0.01 /-0.03$ & $>0.01 /-0.03$ & $>0.01 / 0.19$ \\
\hline $\begin{array}{l}\text { Hip rotation } \\
M I L\end{array}$ & $<0.01 * / 0.57$ & $<0.01 * / 0.59$ & $<0.01 * / 0.62$ & $<0.01 * / 0.62$ & $<0.01 * / 0.62$ & $<0.01 * / 0.62$ & $<0.01 * / 0.59$ & $<0.01 * / 0.56$ \\
\hline $\begin{array}{l}\text { Hip rotation } \\
\text { LIL }\end{array}$ & $>0.01 / 0.28$ & $>0.01 / 0.32$ & $<0.01 * / 0.56$ & $<0.01 * / 0.56$ & $<0.01 * / 0.56$ & $<0.01 \% / 0.56$ & $<0.01 * / 0.56$ & $<0.01 * / 0.56$ \\
\hline $\begin{array}{l}\text { Knee flexion/ } \\
\text { extension MIL }\end{array}$ & $>0.01 /-0.16$ & $>0.01 /-0.06$ & $>0.01 /-0.19$ & $>0.01 /-0.27$ & $>0.01 /-0.11$ & $>0.01 /-0.38$ & $>0.01 /-0.24$ & $>0.01 /-0.24$ \\
\hline $\begin{array}{l}\text { Knee flexion/ } \\
\text { extension LIL }\end{array}$ & $>0.01 / 0.08$ & $>0.01 / 0.08$ & $>0.01 / 0.08$ & $>0.01 / 0.17$ & $>0.01 / 0.08$ & $>0.01 / 0.03$ & $>0.01 /-0.04$ & $>0.01 /-0.01$ \\
\hline Knee abduction/ & $<0.01 / 0.43$ & $<0.01 / 0.43$ & $<0.01 / 0.40$ & $>0.01 / 0.33$ & $<0.01 / 0.43$ & $<0.01 /-0.48$ & $<0.01 / 0.46$ & $<0.01 / 0.48$ \\
\hline
\end{tabular}




\begin{tabular}{|c|c|c|c|c|c|c|c|c|}
\hline adduction MIL & & & & & & & & \\
\hline $\begin{array}{l}\text { Knee abduction/ } \\
\text { adduction LIL }\end{array}$ & $>0.01 / 0.22$ & $>0.01 / 0.22$ & $>0.01 / 0.27$ & $>0.01 / 0.09$ & $>0.01 / 0.25$ & $>0.01 /-0.38$ & $>0.01 / 0.33$ & $<0.01 / 0.43$ \\
\hline $\begin{array}{l}\text { Ankle plantar/ } \\
\text { dorsal flexion } \\
\text { MIL }\end{array}$ & $>0.01 / 0.24$ & $>0.01 / 0.20$ & $>0.01 / 0.14$ & $1 / 0$ & $>0.01 /-0.04$ & $>0.01 / 0.28$ & $>0.01 / 0.28$ & $>0.01 / 0.03$ \\
\hline $\begin{array}{l}\text { Ankle plantar/ } \\
\text { dorsal flexion } \\
\text { LIL }\end{array}$ & $>0.01 / 0.11$ & $>0.01 / 0.14$ & $>0.01 /-0.01$ & $>0.01 /-0.19$ & $>0.01 /-0.19$ & $>0.01 / 0.12$ & $>0.01 / 0.22$ & $>0.01 / 0.09$ \\
\hline Foot tilt MIL & $>0.01 /-0.21$ & $>0.01 /-0.18$ & $>0.01 /-0.16$ & $>0.01 / 0.06$ & $>0.01 / 0.14$ & $>0.01 /-0.04$ & $>0.01 /-0.24$ & $>0.01 /-0.25$ \\
\hline Foot tilt LIL & $>0.01 / 0.32$ & $>0.01 / 0.35$ & $>0.01 / 0.38$ & $>0.01 / 0.30$ & $>0.01 / 0.16$ & $>0.01 / 0.16$ & $>0.01 / 0.04$ & $>0.01 / 0.22$ \\
\hline $\begin{array}{l}\text { Foot progress } \\
\text { MIL }\end{array}$ & $<0.01 / 0.50$ & $<0.01 / 0.49$ & $<0.01 * / 0.56$ & $<0.01 * / 0.57$ & $<0.01 / 0.49$ & $<0.01 / 0.51$ & $<0.01 / 0.44$ & $<0.01 / 0.40$ \\
\hline $\begin{array}{l}\text { Foot progress } \\
\text { LIL }\end{array}$ & $<0.01 * / 0.56$ & $<0.01 / 0.54$ & $<0.01 / 0.46$ & $<0.01 /-0.60$ & $<0.01 /-0.62$ & $>0.01 /-0.38$ & $>0.01 /-0.32$ & $<0.01 /-0.43$ \\
\hline $\begin{array}{l}\text { Thorax tilt } \\
M I S^{*}\end{array}$ & $<0.01 / 0.39$ & $<0.01 / 0.39$ & $<0.01 / 0.39$ & $<0.01 / 0.46$ & $<0.01 / 0.48$ & $<0.01 / 0.48$ & $<0.01 / 0.41$ & $<0.01 / 0.43$ \\
\hline Thorax tilt LIS* & $<0.01 / 0.44$ & $<0.01 / 0.44$ & $<0.01 / 0.43$ & $<0.01 / 0.43$ & $<0.01 / 0.43$ & $<0.01 / 0.44$ & $<0.01 / 0.46$ & $<0.01 / 0.44$ \\
\hline Kinetics & IC & LR & MST & TS & PSW & ISW & MSW & TSW \\
\hline $\begin{array}{l}\text { Hip flexion/ } \\
\text { extension } \\
\text { moment MIL } \\
\end{array}$ & $>0.01 /-0.14$ & $>0.01 /-0.46$ & $>0.01 /-0.35$ & $>0.01 /-0.12$ & $>0.01 / 0.09$ & $>0.01 / 0.19$ & $>0.01 /-0.28$ & $>0.01 /-0.05$ \\
\hline $\begin{array}{l}\text { Hip flexion/ } \\
\text { extension } \\
\text { moment LIL }\end{array}$ & $>0.01 / 0.19$ & $>0.01 /-0.30$ & $>0.01 / 0.04$ & $>0.01 / 0.03$ & $>0.01 /-0.16$ & $>0.01 /-0.09$ & $>0.01 / 0.48$ & $>0.01 / 0.01$ \\
\hline $\begin{array}{l}\text { Hip abduction/ } \\
\text { adduction } \\
\text { moment MIL }\end{array}$ & $>0.01 / 0.22$ & $>0.01 /-0.17$ & $>0.01 /-0.01$ & $>0.01 /-0.04$ & $1 / 0$ & $1 / 0$ & $>0.01 /-0.08$ & $>0.01 /-0.07$ \\
\hline $\begin{array}{l}\text { Hip abduct- } \\
\text { tion/adduction } \\
\text { moment LIL }\end{array}$ & $>0.01 / 0.27$ & $>0.01 /-0.14$ & $>0.01 /-0.09$ & $>0.01 /-0.09$ & $>0.01 / 0.11$ & $>0.01 /-0.06$ & $>0.01 /-0.20$ & $1 / 0$ \\
\hline Hip power MIL & $>0.01 /-0.01$ & $>0.01 / 0.16$ & $>0.01 /-0.08$ & $>0.01 / 0.08$ & $>0.01 / 0.22$ & $>0.01 / 0.21$ & $>0.01 / 0.01$ & $>0.01 / 0.36$ \\
\hline Hip power LIL & $>0.01 / 0.38$ & $>0.01 / 0.23$ & $>0.01 / 0.30$ & $>0.01 /-0.24$ & $>0.01 /-0.30$ & $>0.01 /-0.11$ & $>0.01 / 0.09$ & $>0.01 / 0.25$ \\
\hline $\begin{array}{l}\text { Knee flexion/ } \\
\text { extension } \\
\text { moment MIL }\end{array}$ & $>0.01 /-0.51$ & $>0.01 /-0.17$ & $>0.01 /-0.20$ & $>0.01 / 0.35$ & $>0.01 /-0.11$ & $>0.01 /-0.09$ & $>0.01 / 0.04$ & $>0.01 /-0.35$ \\
\hline $\begin{array}{l}\text { Knee flexion/ } \\
\text { extension } \\
\text { moment LIL }\end{array}$ & $>0.01 /-0.28$ & $>0.01 /-0.52$ & $>0.01 /-0.38$ & $>0.01 / 0.32$ & $>0.01 /-0.25$ & $>0.01 /-0.20$ & $>0.01 / 0.09$ & $>0.01 /-0.08$ \\
\hline $\begin{array}{l}\text { Knee valgus/ } \\
\text { varus moment } \\
\text { MIL }\end{array}$ & $>0.01 / 0.06$ & $>0.01 /-0.20$ & $>0.01 /-0.38$ & $>0.01 / 0.06$ & $>0.01 /-0.12$ & $>0.01 / 0.41$ & $>0.01 / 0.12$ & $>0.01 / 0.24$ \\
\hline $\begin{array}{l}\text { Knee valgus/ } \\
\text { varus moment } \\
\text { LIL }\end{array}$ & $>0.01 /-0.36$ & $>0.01 /-0.01$ & $>0.01 /-0.03$ & $>0.01 /-0.03$ & $>0.01 / 0.19$ & $>0.01 /-0.14$ & $>0.01 /-0.33$ & $>0.01 /-0.22$ \\
\hline $\begin{array}{l}\text { Knee power } \\
\text { MIL }\end{array}$ & $>0.01 /-0.16$ & $>0.01 / 0.14$ & $>0.01 / 0.06$ & $>0.01 /-0.14$ & $>0.01 /-0.13$ & $>0.01 /-0.21$ & $>0.01 / 0.04$ & $>0.01 / 0.25$ \\
\hline Knee power LIL & $>0.01 /-0.01$ & $>0.01 /-0.05$ & $>0.01 /-0.14$ & $>0.01 /-0.28$ & $>0.01 / 0.29$ & $>0.01 / 0.36$ & $>0.01 / 0.01$ & $>0.01 /-0.14$ \\
\hline
\end{tabular}




\begin{tabular}{|c|c|c|c|c|c|c|c|c|}
\hline $\begin{array}{l}\text { Ankle flexion/ } \\
\text { extension } \\
\text { moment MIL }\end{array}$ & $>0.01 /-0.08$ & $>0.01 / 0.04$ & $>0.01 /-0.12$ & $>0.01 /-0.20$ & $>0.01 / 0.16$ & $>0.01 / 0.27$ & $>0.01 /-0.20$ & $>0.01 /-0.28$ \\
\hline $\begin{array}{l}\text { Ankle flexion/ } \\
\text { extension } \\
\text { moment LIL }\end{array}$ & $>0.01 / 0.09$ & $>0.01 / 0.27$ & $>0.01 /-0.01$ & $>0.01 /-0.25$ & $>0.01 / 0.03$ & $>0.01 / 0.11$ & $>0.01 / 0.09$ & $>0.01 /-0.19$ \\
\hline $\begin{array}{l}\text { Ankle power } \\
\text { MIL }\end{array}$ & $>0.01 /-0.06$ & $>0.01 /-0.41$ & $>0.01 /-0.12$ & $>0.01 /-0.17$ & $>0.01 /-0.03$ & $>0.01 /-0.13$ & $>0.01 /-0.14$ & $>0.01 /-0.09$ \\
\hline $\begin{array}{l}\text { Ankle power } \\
\text { LIL }\end{array}$ & $>0.01 / 0.22$ & $>0.01 /-0.01$ & $>0.01 / 0.12$ & $>0.01 /-0.08$ & $>0.01 /-0.47$ & $>0.01 / 0.15$ & $>0.01 /-0.14$ & $>0.01 / 0.04$ \\
\hline GRF XMIL & $>0.01 /-0.09$ & $>0.01 /-0.30$ & $>0.01 /-0.16$ & $>0.01 /-0.30$ & $>0.01 / 0.28$ & & & \\
\hline GRF X LIL & $1 / 0$ & $>0.01 /-0.32$ & $>0.01 /-0.28$ & $>0.01 /-0.24$ & $>0.01 /-0.25$ & & & \\
\hline GRF Y MIL & $>0.01 /-0.16$ & $>0.01 /-0.16$ & $>0.01 / 0.24$ & $>0.01 / 0.22$ & $>0.01 / 0.17$ & & & \\
\hline GRF Y LIL & $>0.01 /-0.03$ & $>0.01 / 0.14$ & $>0.01 / 0.06$ & $>0.01 /-0.14$ & $>0.01 /-0.03$ & & & \\
\hline GRF Z MIL & $>0.01 / 0.14$ & $>0.01 / 0.12$ & $>0.01 /-0.01$ & $>0.01 / 0.12$ & $>0.01 / 0.11$ & & & \\
\hline GRF Z LIL & $>0.01 / 0.05$ & $>0.01 / 0.23$ & $>0.01 / 0.17$ & $>0.01 /-0.01$ & $>0.01 /-0.49$ & & & \\
\hline COM MIL & $>0.01 / 0.05$ & $>0.01 / 0.01$ & $>0.01 / 0.24$ & $>0.01 / 0.09$ & $>0.01 / 0.03$ & & & \\
\hline COM LIL & $>0.01 / 0.16$ & $>0.01 / 0.14$ & $>0.01 / 0.08$ & $>0.01 / 0.06$ & $1 / 0$ & & & \\
\hline COP X MIL & $>0.01 / 0.12$ & $>0.01 /-0.03$ & $1 / 0$ & $>0.01 /-0.24$ & $>0.01 /-0.03$ & & & \\
\hline COP X LIL & $>0.01 / 0.03$ & $>0.01 / 0.03$ & $>0.01 / 0.04$ & $>0.01 / 0.01$ & $>0.01 /-0.02$ & & & \\
\hline COP Y MIL & $>0.01 /-0.32$ & $>0.01 /-0.38$ & $>0.01 /-0.30$ & $>0.01 /-0.22$ & $>0.01 /-0.24$ & & & \\
\hline COP Y LIL & $>0.01 / 0.18$ & $>0.01 /-0.11$ & $>0.01 /-0.30$ & $>0.01 /-0.22$ & $>0.01 /-0.25$ & & & \\
\hline
\end{tabular}

SEMG (surface electromyography); BF (biceps femoris); RF (rectus femoris); TA (tibialis anterior); MG (medial gastrocnemius); MIL (More impaired limb); LIL (Less impaired limb); IC (Initial contact); LR (Loading response); MST (Midstance); TS (Terminal stance); PSW (Preswing); ISW (Initial swing); MSW (Midswing); TSW (Terminal swing).

Table 2. Table shows an overview of Wilcoxon sign rank tests for spatiotemporal parameters with corrected $p$-value and median values. Statistically significant results $(p \leq 0.01)$ are bolded and marked with an asterisk $(*)$.

\begin{tabular}{|c|c|c|c|c|c|c|}
\hline $\begin{array}{l}\text { Spatiotemporal } \\
\text { parameters }\end{array}$ & $\begin{array}{c}\text { Median MIL } \\
\text { pre } \\
\end{array}$ & $\begin{array}{c}\text { Median MIL } \\
\text { post } \\
\end{array}$ & $\begin{array}{c}\text { MIL p-value/ } \\
\text { effect size }\end{array}$ & $\begin{array}{c}\text { Median LIL } \\
\text { pre } \\
\end{array}$ & $\begin{array}{c}\text { Median LIL } \\
\text { post } \\
\end{array}$ & $\begin{array}{c}\text { LIL p-value/ } \\
\text { effect size }\end{array}$ \\
\hline Cadence (steps/min) & 109.04 & 111.57 & $<0.01 * / 0.62$ & 109.55 & 112.45 & $<0.01 * / 0.61$ \\
\hline Double support (s) & 0.36 & 0.34 & $<0.01 * / 0.63$ & 0.37 & 0.33 & $<0.01 * / 0.62$ \\
\hline Foot off (\%) & 64.35 & 64.19 & $<0.01 / 0.52$ & 65.04 & 64.97 & $<0.01 * / 0.61$ \\
\hline Opposite foot contact (\%) & 48.48 & 48.91 & $>0.01 / 0.04$ & 51.67 & 51.18 & $>0.01 / 0.04$ \\
\hline Opposite foot off (\%) & 13.87 & 13.59 & $>0.01 / 0.04$ & 16.66 & 15.08 & $>0.01 / 0.04$ \\
\hline Single support (s) & 0.39 & 0.39 & $>0.01 / 0.06$ & 0.39 & 0.39 & $>0.01 / 0.04$ \\
\hline Step length $(m)$ & 0.43 & 0.46 & $<0.01 * / 0.61$ & 0.43 & 0.45 & $<0.01 * / 0.60$ \\
\hline Step time $(s)$ & 0.6 & 0.58 & $>0.01 / 0.05$ & 0.55 & 0.54 & $>0.01 / 0.05$ \\
\hline Step width (m) & 0.11 & 0.12 & $>0.01 / 0.05$ & 0.11 & 0.12 & $>0.01 / 0.05$ \\
\hline Stride length (m) & 0.87 & 0.85 & $<0.01 / 0.52$ & 0.87 & 0.83 & $<0.01 / 0.52$ \\
\hline Stride time (s) & 1.16 & 1.13 & $<0.01 * / 0.59$ & 1.16 & 1.12 & $<0.01 * / 0.59$ \\
\hline Walking speed $(\mathrm{m} / \mathrm{s})$ & 0.8 & 0.87 & $<0.01 / 0.52$ & 0.81 & 0.86 & $<0.01 / 0.52$ \\
\hline
\end{tabular}

MIL (More impaired limb); LIL (Less impaired limb). 
Table 3. Table shows an overview of median values for cross-correlation coefficients for CGA variables that were statistically significant $(p \leq 0.01)$. This optimization was done due to an extensive amount of variables.

\begin{tabular}{|c|c|c|c|c|c|}
\hline \multirow{2}{*}{$E M G$} & \multicolumn{2}{|c|}{ Median of cross-correlation } & \multirow{2}{*}{ Kinematics } & \multicolumn{2}{|c|}{ Median of cross-correlation } \\
\hline & Before & After & & Before & After \\
\hline BF MIL MST & 1.57E-07 & $9.74 \mathrm{E}-08$ & Hip rotation MIL IC & -104.813168 & -35.353415 \\
\hline BF MIL TS & 4.19E-08 & $2.18 \mathrm{E}-08$ & Hip rotation MIL LR & -407.577837 & -131.401828 \\
\hline BF LIL TS & $5.09 \mathrm{E}-08$ & 2.63E-08 & Hip rotation MIL MST & -131.317739 & -60.436948 \\
\hline BF MIL PSW & 1.07E-08 & $5.36 \mathrm{E}-09$ & Hip rotation LIL MST & -56.233293 & -11.707981 \\
\hline BF LIL ISW & $1.52 \mathrm{E}-08$ & $1.10 \mathrm{E}-08$ & Hip rotation MIL TS & -225.096715 & -85.055612 \\
\hline BF MIL TSW & $1.25 \mathrm{E}-07$ & $7.77 \mathrm{E}-08$ & Hip rotation LIL TS & -80.912522 & -50.977734 \\
\hline BF LIL TSW & 1.33E-07 & $9.78 \mathrm{E}-08$ & Hip rotation MIL PSW & -198.098633 & -91.203742 \\
\hline RF MIL IC & $1.85 \mathrm{E}-08$ & $1.07 \mathrm{E}-08$ & Hip rotation LIL PSW & -91.927671 & -61.537886 \\
\hline RF LIL IC & $1.54 \mathrm{E}-08$ & $1.15 \mathrm{E}-08$ & Hip rotation MIL ISW & -100.699038 & -35.898468 \\
\hline$R F M I L L R$ & $5.83 \mathrm{E}-08$ & $3.50 \mathrm{E}-08$ & Hip rotation LIL ISW & -46.63385 & $-\mathbf{3 3 . 8 9 8 3 3 7}$ \\
\hline$R F$ LIL LR & 5.23E-08 & $4.10 \mathrm{E}-08$ & Hip rotation MIL MSW & 568.936576 & 370.747144 \\
\hline RF MIL MST & $8.21 E-08$ & $4.15 \mathrm{E}-08$ & Hip rotation LIL MSW & 359.984115 & 161.270348 \\
\hline$R F$ LIL MST & 7.05E-08 & $5.33 \mathrm{E}-08$ & Hip rotation MIL TSW & 256.714316 & 106.666952 \\
\hline$R F M I L T S$ & $2.30 \mathrm{E}-08$ & $1.31 \mathrm{E}-08$ & Hip rotation LIL TSW & 166.934347 & 12.34891 \\
\hline RF LIL TS & 2.07E-08 & $1.37 \mathrm{E}-08$ & Foot progress LIL IC & -53.74 & 7.65 \\
\hline$R F M I L P S W$ & 1.13E-08 & $6.67 \mathrm{E}-09$ & Foot progress MIL MST & -80.71 & 14.77 \\
\hline$R F M I L M S W$ & $1.44 \mathrm{E}-08$ & $9.01 \mathrm{E}-09$ & Foot progress MIL TS & -81.67 & 265.39 \\
\hline$R F M I L T S W$ & $1.92 \mathrm{E}-08$ & $1.23 \mathrm{E}-08$ & & & \\
\hline TA LIL IC & $1.45 \mathrm{E}-07$ & 8.02E-08 & & & \\
\hline$T A L I L L R$ & 4.23E-07 & $2.14 \mathrm{E}-07$ & & & \\
\hline TA LIL MST & $2.78 \mathrm{E}-07$ & $1.57 \mathrm{E}-07$ & & & \\
\hline TA LIL TS & $5.93 E-08$ & $4.08 \mathrm{E}-08$ & & & \\
\hline
\end{tabular}

SEMG (surface electromyography); BF (biceps femoris); RF (rectus femoris); TA (tibialis anterior); MG (medial gastrocnemius); MIL (More impaired limb); LIL (Less impaired limb); IC (Initial contact); LR (Loading response); MST (Midstance); TS (Terminal stance); PSW (Preswing); ISW (Initial swing); MSW (Midswing); TSW (Terminal swing).

\section{Discussion}

\section{Interpretation of sEMG results}

Since active training seems to be more effective than passive training for motor learning and cortical reorganization in central motor impairments, RAGT likely improved muscle activation of children with $\mathrm{CP}$ due to active training performed with a high-repetitionrate of guided movements (Meyer-Heim et al. 2009, Bonikowski et al. 2012, Aurich-Schuler 2017). Although this research study did not explore spasticity in children with $\mathrm{CP}$, it could be one of the supportive explanations for why RAGT led to the decrease of muscle activity. Cyclic motion has been reported to be effective in decreasing spasticity in stroke patients (Monaghan 2017). In children with $\mathrm{CP}$, the RF muscle tends to be shortened and spastic. This leads to hip joint flexion contractures that do not allow active and controlled knee extension during the stance phase (Foran 2005, Katz 1989, Schuler et al. 2011, Schuler et al. 2013). BF is typically weakened due to the dominant activity of the antagonist RF. Despite that in some children with CP this muscle can be spastic (Goldberg et al. 2012). Although our findings showed the bilaterally decreased activity of both muscles (Bonikowski et al. 2012) almost across all gait cycle phases, it is worth elaborating on increased number of treatments (e.g. up to 40) in a longer period of time such as in the study of Verazaluce-Rodriguez et al. (2014) to explore whether it is possible to induce even more physiological muscle activity. Children with $\mathrm{CP}$ often have spastic calf muscles, foot deformities and difficulties performing dorsiflexion and foot inversion resulting in a lack of TA activity (Brunner et al. 2008). In this study, TA muscle was active post-intervention during swing up to the loading response phases as previously shown in studies performed on healthy subjects (Brutsch 
et al. 2010, Schuler et al. 2013, Schuler et al. 2017). Thus, our findings further support that RAGT can enhance the physiological activity of TA, although the ankle joint during RAGT is only passively positioned to the neutral position (Colombo 2000). MG is a biarticular muscle often considered the main contributor to abnormal gait patterns as it causes Achilles tendon shortening which can result in contracture and foot deformities (Patikas et al. 2007, Perry 2010, Stewart et al. 2010). Therefore, if the MG muscle is influenced, either by stretching, relaxation, or positioning the affected joint in the neutral position, it can contribute to the increased range of motion in the ankle joint, as well as it can reciprocally allow the activation of the antagonist TA muscle (Katz et al. 1989, Colombo et al. 2000, Colombo et al. 2005). This is an interesting finding because it indicates that perhaps the active support and active movement of the proximal musculature may help encourage a similar adaptation in the distal musculature despite a lack of active support (Radziminska et al. 2012, Vrečar et al. 2013).

\section{Interpretation of joint kinematics and kinetics results}

Despite the lack of studies that explored the effect of RAGT on joint kinematics and kinetics in children with CP (Druzbicki et al. 2013, Schuler et al. 2017, Wallard et al. 2018), to the best of the authors' knowledge, this is the first study reporting on changes that followed RAGT in hip joint rotations. It is assumed that RAGT likely decreased internal hip joint rotations due to a high repetition rate of guided movements in joint centered position of the pelvis and lower limbs (Kolár 2002, Žarković and Šorfová 2017). In conditions with impaired motor control, such as in children with $\mathrm{CP}$, joints are in a so-called decentralized position that also contributes to improper muscle function. The centered joint position allows for optimal loading of the joint in both static and dynamic conditions, as well as it enhances physiological muscle patterns. This is an interesting finding because it indicates that the combination of taskspecific guided movements in a high-repetition-rate, and centered position of joints resulted in a decreased pathological internal hip joint rotations in ambulatory children with CP. Although RAGT allows very detailed adjustment of gait parameters, it has a fixed strategy to control the motion trajectory of the robotic orthoses and pelvis, which are limited to one degree of freedom in the sagittal plane. Indeed, it is the absence of lateral weight shift to the standing limb, rotational pelvic movements and kinematic variability that may be the main causes of therapy irresponsiveness in lower limb kinematic and kinetic variables. This is also supported by studies that have found that lower limb and pelvic kinematic variability could facilitate the transfer of motor skills from robotic therapy to over-ground walking (Reinkensmeyer et al. 2006, Koopman et al. 2013, Wu et al. 2017).

\section{Interpretation of spatiotemporal parameters}

Definition of spatiotemporal parameters allows for an objective definition of where, when, how long, and how rapidly the individual is in contact with the ground (Perry 2010, Baker 2013, Armand et al. 2016). In this research study, the most important changes were observed in increased cadence and step length, and a decrease in time needed for double support and stride. This could potentially contribute to more economic gait pattern in ambulatory children with $\mathrm{CP}$ who need to walk farther distances. Similar findings were reported by Meyer-Heim et al. 2007, Knecht et al. 2010, Beretta et al. 2015.

\section{Conclusions}

Findings suggest that RAGT as monotherapy can contribute to muscle reeducation and improvement of hip joint motion range in ambulatory children with $\mathrm{CP}$. This is the first research study that extended the explanation of RAGT by centered joint position. Authors are aware of study limitations such as the small sample size, lack of control group and long-term follow-up data. For that, there is no tendency to generalize the study results to a wider spectrum of the $\mathrm{CP}$ population. However, this research study provides a foundation on which future studies can be built as RAGT should be investigated over longer periods in different populations to further determine its effectiveness.

\section{Conflict of Interest}

There is no conflict of interest.

\section{Acknowledgements}

This study was funded by university student support FTVS SVV 2020-2022-260599 and institutional support "Progress Q41 2017-2021 biological aspects of the investigation of human movement" from Charles University, Prague, Czech Republic. Authors state that there is no conflict of interest. 


\section{References}

ARMAND S, DECOULON G, BONNEFOY-MAZURE A: Gait analysis in children with cerebral palsy. EFORT Open Rev 1: 448-460, 2016. https://doi.org/10.1302/2058-5241.1.000052

BAKER RW: Measuring Walking: A Handbook of Clinical Gait Analysis. New Jersey: Wiley, 2013, 246 p.

BAX M, GOLDSTEIN M, ROSENBAUM P, LEVITON A, PANETH N: Proposed definition and classification of cerebral palsy. Dev Med Child Neurol 47: 571-576, 2005. https://doi.org/10.1017/S001216220500112X

BOJANIC DM, PETROVACKI-BALJ BD, JORGOVANOVIC ND, ILIC VR: Quantification of dynamic EMG patterns during gait in children with cerebral palsy. J Neurosci Methods 15: 325-331, 2011. https://doi.org/10.1016/j.jneumeth.2011.04.030

BONIKOWSKI M, MROZEK P: Changes in surface EMG patterns in children with cerebral palsy during robotic gait training. Gait Posture 36: 70, 2012. https://doi.org/10.1016/j.gaitpost.2011.10.300

BRUNNER J, ROMKES J: Abnormal EMG muscle activity during gait in patients without neurological disorders. Gait Posture 27: 399-407, 2008. https://doi.org/10.1016/j.gaitpost.2007.05.009

BRÜTSCH K, SCHULER T, KOENIG A, ZIMMERLI L, MERILLAR S, LUNENBURGER L, RIENER R, JANCKE L, MEYER-HEIM A: Influence of virtual reality soccer game on walking performance in robotic assisted gait training for children. J Neuroeng Rehabil 7: 493-499, 2010. https://doi.org/10.1186/1743-0003-7-15

BURDEN A, ARTLETT R: Normalisation of EMG amplitude: an evaluation and comparison of old and new methods. Med Eng Phys 21: 247-257, 1999. https://doi.org/10.1016/S1350-4533(99)00054-5

BURDEN AM, TREW M, BALTZOPOULOS V: Normalisation of gait EMGs: a re-examination. J Electromyogr Kinesiol 13: 519-532, 2003. https://doi.org/10.1016/S1050-6411(03)00082-8

COHEN J: Differences between correlation coefficients. In: Statistical Power Analysis for the Behavioral Sciences. COHEN J (ed.), Lawrence Erlbaum Associates, New York, 1988, pp 109-139. https://doi.org/10.1016/B978-012-179060-8.50009-8

COLUMBO G, JOERG M, SCHREIER R, DIETZ V: Treadmill training of paraplegic patients using a robotic orthosis. J Rehabil Res Dev 37: 693-700, 2000.

DAVIS RB, ÕUNPUU S, TYBURSKI D, GAGE JR: A gait analysis data collection and reduction technique. Human Mov Sci 10: 575-587, 1991. https://doi.org/10.1016/0167-9457(91)90046-Z

DIXON CP, STEBBINS J, THEOLOGIS T, ZAVATSKY AB: Ground reaction forces and lower-limb joint kinetics of turning gait in typically developing children. J Biomech 47: 3726-3733, 2014. https://doi.org/10.1016/j.jbiomech.2014.09.011

DRUZBICKI M, RUSEK W, SNELA S, DUDEK J, SZCZEPANIK M, ZAK E: Functional effects of robotic-assisted locomotor treadmill therapy in children with cerebral palsy. J Rehabil Med 45: 358-363, 2013. https://doi.org/10.2340/16501977-1114

FLUX E, VAN DER KROGT MM, CAPPA P, PETRARCA M, DESLOOVERE K, HARLAN J: The human body model versus conventional gait models for kinematic gait analysis in children with cerebral palsy. Human Mov Sci 70: 102585, 2020. https://doi.org/10.1016/j.humov.2020.102585

FORAN JR, STEINMAN S, BARASH I, CHAMBERS HG, LIEBER RL: Structural and mechanical alterations in spastic skeletal muscle. Dev Med Child Neurol 47: 713-717, 2005. https://doi.org/10.1017/S0012162205001465

FOWLER EG, STAUDT LA, GREENBERG MB, OPPENHEIM WL: Selective Control Assessment of the Lower Extremity (SCALE): Development, validation, and interrater reliability of a clinical tool for patients with cerebral palsy. Dev Med Child Neurol 51: 607-614, 2009. https://doi.org/10.1111/j.1469-8749.2008.03186.x

FUNG J, BARBEAU H: A dynamic EMG profile index to quantify muscular activation disorder in spastic paretic gait. Electroencephalogr Clin Neurophysiol 73: 233-244, 1989. https://doi.org/10.1016/0013-4694(89)90124-7

GOLDBERG EJ, FOWLER EG, OPPENHEIM WL: Case reports: The influence of selective voluntary motor control on gait after hamstring lengthening surgery. Clin Orthop Relat Res 470: 1320-1326, 2012. https://doi.org/10.1007/s11999-011-2028-2 
GORDON AM, MAGILL RA: Motor learning: Application of principles to pediatric rehabilitation. In: Physical Therapy for Children. CAMPBELL SK, PALISANO RJ, ORLIN MN (eds), MO Elsevier, St. Louis, 2012, pp 151-174.

HEMAYATTALAB R, ARABAMERI E, POURAZAR M, DEHESTANI ARDAKANI M, KA-SHEFI M: Effects of self-controlled feedback on learning of a throwing task in children with spastic hemiplegic cerebral palsy. Res Dev Disabil 34: 2884-2889, 2013. https://doi.org/10.1016/j.ridd.2013.05.008

HEMAYATTALAB R, RASHIDI ROSTAMI L: Effects of frequency of feedback on the learning of motor skill in individuals with cerebral palsy. Res Dev Disabil 31: 212-217, 2010. https://doi.org/10.1016/j.ridd.2009.09.002

HERMENS HJ, FRERIKS B, MERLETTI R, STEGEMAN D, BLOK J, RAU G, DISSELHORST-KLUG C, HÄGG G: SENIAM 8: European Recommendations for Surface Electromyography. Roessingh Research and Development, 1999, $112 \mathrm{p}$.

HOF AL, ELZINGA H, GRIMMIUS W, HALBERTSMA JP: Detection of non-standard EMG profiles in walking. Gait Posture 21: 171-177, 2005. https://doi.org/10.1016/j.gaitpost.2004.01.015

KATZ RT, RYMER WZ: Spastic hypertonia: mechanisms and measurement. Arch Phys Med Rehabil 70: 144-155, 1989.

KNECHT B: Robotic-assisted treadmill therapy improves walking and standing performance in children and adolescents with cerebral palsy. Eur J Paediatr Neurol 14: 496-502, 2010. https://doi.org/10.1016/j.ejpn.2010.01.002

KOLÁŘ P: Vadné držení těla z pohledu posturální ontogeneze. Pediatrie pro praxi 3: 106-109, 2002.

KOOPMAN B, VAN ASSELDONK EH, VAN DER KOOIJ H: Selective control of gait sub-tasks in robotic gait training: foot clearance support in stroke survivors with a powered exo-skeleton. J NeuroEngineering Rehabil 10: 3, 2013. https://doi.org/10.1186/1743-0003-10-3

JANDA V, PAVLU D: Goniometrie. Brno: Institut pro dalsi vzdelavani pracovniku ve zdravotnictvi; 1993.

MACWILLIAMS BA, COWLEY M, NICHOLSON DE: Foot kinematics and kinetics during adolescent gait. Gait Posture 17: 214-224, 2003. https://doi.org/10.1016/S0966-6362(02)00103-0

MEYER-HEIM A, BORGGRAEFFE I, AMMANN-REIFFER C: Feasibility of robotic-assisted locomotor training in children with central gait impairment. Dev Med Child Neurol 49: 900-906, 2007. https://doi.org/10.1111/j.1469-8749.2007.00900.x

MONAGHAN K, HORGAN F, BLAKE C, CORNALL C, HICKEY PPM, LYONS BE, LANG-HORNE P: Physical treatment interventions for managing spasticity after stroke. Cochrane Dat Syst Rev 2: 1-24, 2017. https://doi.org/10.1002/14651858.CD009188.pub2

NOVAK I: Evidence-based diagnosis, health care, and rehabilitation for children with cerebral palsy. J Child Neurol 29 : 1141-1156, 2014. https://doi.org/10.1177/0883073814535503

PANTELIADIS CP, STRASSBURG HM: History and terminology, classification, incidence and prevalence, aetiology, neuropathology of cerebral palsy, clinical characteristics. In: Cerebral Palsy: Principles and Management. PANTELIADIS CP, STRASSBURG HM (eds), Georg Thieme Verlag Stuttgart-New York, 2004, pp 5-60.

PATIKAS D, WOLF SI, SCHUSTER W, ARMBRUST P, DREHER D, DODERLEIN L: Electromyographic patterns in children with cerebral palsy: Do they change after surgery? Gait Posture 26: 362-371, 2007. https://doi.org/10.1016/j.gaitpost.2006.10.012

PERRY J, BURNFIELD JM: Pathological gait. In: Gait Analysis - Normal and Pathological Function. PERRY J, BURNFIELD JM (eds), Slack Incorporated, New Jersey, 2010, pp 163-237.

RADZIMINSKA A, SROKOWSKI G, BULATOWICZ I, KAZMIERCZAK U, STROJEK K, BAUMGART M, ZUKOW W: Assessment of the PNF method influence on gait parameters improvement in persons with cerebral palsy. Humanities dimension of medicine and physiotherapy. Bydgoszcz: University of Health Sciences, 57-74, 2012.

REINKENSMEYER DJ, AOYAGI D, EMKEN JL, GALVEZ JA, ICHI-NOSE W, KERDANYAN G, MANEEKOBKUNWONG S, MINAKATA K, NESSLER JA, WEBER R, ROY RR, DE LEON R, BOBROW JE, HARKEMA SJ, EDGERTON VR: Tools for understanding and optimizing robotic gait training. J Rehabil Res Dev 43: 657-670, 2006. https://doi.org/10.1682/JRRD.2005.04.0073 
RIENER R, LÜNENBURGER L, JEZERNIK S, ANDERSCHITZ M, COLOMBO G, DIETZ V: Patient-cooperative strategies for robot-aided treadmill training: first experimental results. IEEE Trans Neural Syst Rehabil Eng 13: 380-394, 2005. https://doi.org/10.1109/TNSRE.2005.848628

SANKAR CH, MUNDKUR N: Cerebral palsy-definition, classification, etiology and early diagnosis. Indian J Pediatr 72: 865-868, 2005. https://doi.org/10.1007/BF02731117

SCHULER T, BRÜTSCH K, MÜLLER R, VAN HEDEL HJ, MEYER-HEIM A: Virtual realities as motivational tools for robotic assisted gait training in children: A surface electromyography study. Neurorehabilitation 28: 401-411, 2011. https://doi.org/10.3233/NRE-2011-0670

SCHULER T, MÜLLER R, VAN HEDEL HJ: Leg surface electromyography patterns in children with neuro-orthopedic disorders walking on a treadmill unassisted and assisted by a robot with and without encouragement. J Neuroeng Rehabil 10: 78, 2013. https://doi.org/10.1186/1743-0003-10-78

SCHULER T, GROB F, VAN HEDEL HJ, LABRUYERE R: Can Lokomat therapy with children and adolescents be improved? An adaptive clinical pilot trial comparing Guidance force, Path control, and FreeD. J Neuroeng Rehabil 14: 76, 2017. https://doi.org/10.1186/s12984-017-0287-1

STEWART C, SHORTLAND AP: The biomechanics of pathological gait - from muscle to movement. Acta Bioeng Biomech 12: 3-12, 2010.

SUTHERLAND DH, DAVIDS JR: Common gait abnormalities of the knee in cerebral palsy. Clin Orthop Relat Res 288: 139-147, 1993. https://doi.org/10.1097/00003086-199303000-00018

SYCZEWSKA M, ŚWIĘCICKA A: Are electromyographic patterns during gait related to abnormality level of the gait in patients with spastic cerebral palsy? Acta Bioeng Biomech 18: 91-96, 2016.

VERAZALUCE-RODRÍGUEZ PR, RODRÍGUEZ-MARTÍNEZ P, NERI-GÁMEZ S, HERNÁNDEZ-AQUINO RM: Evolution of gait in patients with cerebral palsy and assisted movement by training with robotic help equipment. Rehabilitación 48: 3-8, 2014. https://doi.org/10.1016/j.rh.2013.04.006

VREČAR I, MAJDIČ N, JEMEC I, DAMJAN H: Changes in passive range of motion of joints of the lower limbs in children with cerebral palsy after an intense training program on the Lokomat Rehabilitacija 12: 38-45, 2013.

WALLARD L, DIETRICH G, KERLIRZIN Y, BREDIN J: Effect of robotic-assisted gait rehabilitation on dynamic equilibrium control in the gait of children with cerebral palsy. Gait Posture 60: 55-60, 2018. https://doi.org/10.1016/j.gaitpost.2017.11.007

WINTER D: Biomechanics and Motor Control of Human Movement. John Wiley \& Sons, New Jersey, 2009,384 p. https://doi.org/10.1002/9780470549148

WU M, KIM J, GAEBLER-SPIRA DJ, SCHMIT BD, ARORA P: Robotic resistance treadmill training improves locomotor function in children with cerebral palsy: A randomized controlled pilot study. Arch Phys Med Rehabil 98: 2126-2133, 2017. https://doi.org/10.1016/j.apmr.2017.04.022

ZARKOVIC D, SORFOVA M: Neurobiomechanical Aspects of Robotic Assisted Gait Training. Rehab Fyzik Lek 24: 43-49, 2017.

ZARKOVIC D, SORFOVA M, TUFANO JJ, KUTILEK P, VITECKOVA S, GROLEGER-SRSEN K, RAVNIK D: Effect of robot-assisted gait training on selective voluntary motor control in ambulatory children with cerebral palsy. Indian Pediatr 57: 964-966, 2020. https://doi.org/10.1007/s13312-020-2005-5 